\title{
Nano-tribological investigations of carbon overcoats: correlation with Raman spectra
}

\author{
Chung-Jen Lu ${ }^{a}$, D.B. Bogy ${ }^{a}$, Stephen S. Rosenblum ${ }^{b}$, Glenn J. Tessmer ${ }^{c}$ \\ a Computer Mechanics Laboratory, Department of Mechanical Engineering, University of California at Berkeley, Berkeley, CA 94720, USA \\ ${ }^{\mathrm{b}}$ Applied Electronics Center, Kobe Steel USA, Inc., Palo Alto, CA 94304, USA \\ ' Electronic Materials Center, Kobe Steel USA, Inc., Research Triangle Park, NC 27709, USA
}

Received 19 July 1994; accepted 6 June 1995

\begin{abstract}
Nano-indentation and nano-wear tests were performed on carbon overcoats, which were deposited under different conditions on thin film magnetic disks, using the point contact microscope (PCM). Two failure mechanisms of the overcoats during the nano-wear test are described: one is the wear of the carbon overcoat as measured by the wear depth; the other is the damage at the overcoat-substrate interface as indicated by the critical cycle. The hardness of the carbon overcoat correlates well with the wear resistance. Also a certain relation between the Raman spectra and mechanical properties of carbon overcoats is found. However, it is shown that the Raman spectra can not be used as the sole indicator of the wear resistance of carbon overcoats.
\end{abstract}

Keywords: Tribology; Carbon; Raman scattering; Nanostructures

\section{Introduction}

Amorphous carbon films have been widely used as protective overcoats on magnetic recording disks. Carbon films prepared by different techniques, e.g. sputtering, evaporation and plasma-assisted chemical vapor deposition, have different physical properties that depend on the deposition techniques as well as the deposition conditions [1]. Owing to their ability to form different bonding configurations, carbon films can have a wide span of hardness values, as well as friction and wear properties. Improvement of the tribological performance of carbon overcoats can be achieved by having a more diamond-like carbon $\left(\mathrm{sp}^{3}\right)$ structure. In order to optimize the deposition process, it is important to find the relationship between the intrinsic properties and the deposition conditions. Raman spectroscopy has proven to be an effective method for studying the micro structure of carbon films [24]. Therefore, Raman spectroscopy can be used to finely tune the deposition conditions if there exists a correlation between the mechanical properties and the Raman spectra of carbon films.

The Raman spectrum of diamond consists of a single line at $1332 \mathrm{~cm}^{-1}$, while that of large single-crystal graphite has a single peak at $1580 \mathrm{~cm}^{-1}$, called the G-band. For amorphous carbon, the absence of long-range order shifts the Gband position and creates a new band around $1360 \mathrm{~cm}^{-1}$, called the D-band. The frequencies of the $G$ and $D$ bands have been correlated with $\mathrm{sp}^{3} / \mathrm{sp}^{2}$ ratios [5] and crystal size [4]. Dines et al. [1] found that the intensity of the Dband grows with decreasing crystal size. Studies of graphite materials with Raman spectroscopy show a linear relationship between $I(\mathrm{D}) / I(\mathrm{G})$ and $1 / L_{\mathrm{a}}$, where the crystallite size $L_{\mathrm{a}}$ is obtained by $\mathrm{X}$-ray diffraction line broadening $[6,7]$.

Many investigators have studied the relation between Raman spectra and wear resistance of carbon overcoats of thin film media. Marchon et al. [8] found that the tribochemical wear rate, as estimated by the rate of friction buildup, is correlated to the G-band position. Abrasive wear as measured by the number of scratches is best correlated to the $I(\mathrm{D}) / I(\mathrm{G})$ intensity ratio. Lauer and DuPlessis [9], who performed pin-on-disk tests on carbon overcoats, correlated the wear rate to the separation between the $G$ and $D$ bands instead of either the G-band position or $I(\mathrm{D}) / I(\mathrm{G})$ ratio. There the wear rate was calculated from the distance the pin moved before it penetrated the overcoat, as indicated by a sharp increase in the reflectivity of the wear track. They also found that the substrate temperature during deposition is an important factor, i.e. the correlation mentioned above only exists between carbon overcoats sputtered at the same substrate temperature. On the other hand, Lee et al. [10] found that soft and hard amorphous carbon films may have similar 
Table 1

Deposition conditions for samples K1-K8

\begin{tabular}{llrlr}
\hline Sample & Sputter power $(\mathrm{W})$ & Substrate temperature $\left({ }^{\circ} \mathrm{C}\right)$ & Sputter pressure (mTorr) & Substrate d.c. bias $(\mathrm{V})$ \\
\hline $\mathrm{K} 1$ & 1000 & 250 & 3 & -100 \\
$\mathrm{~K} 2$ & 1000 & 250 & 1.5 & -100 \\
$\mathrm{~K} 3$ & 1000 & 60 & 3 & -100 \\
$\mathrm{~K} 4$ & 500 & 250 & 1.5 & 0 \\
$\mathrm{~K} 5$ & 1000 & 60 & 1.5 & 0 \\
$\mathrm{~K} 6$ & 500 & 60 & 3 & 0 \\
$\mathrm{~K} 7$ & 500 & 250 & 1 & -100 \\
$\mathrm{~K} 8$ & 500 & 60 & & 0 \\
\hline
\end{tabular}

Raman spectra. This implies that the Raman spectra alone cannot be used to distinguish different carbon films.

In the present study, our goal is to determine if the mechanical properties of the carbon films can be deduced from the Raman spectrum directly. Carbon films of about $30 \mathrm{~nm}$ thick were deposited under different conditions to provide a wide range of Raman spectra. The point contact microscope (PCM), which permits accurate characterizations of the ultrathin carbon film without the influence from the substrate [11-13], was used to measure several mechanical properties including hardness, wear resistance and film-substrate adhesion. Different mechanisms during the nano-wear test are described.

\section{Samples}

Eight thin film magnetic disks, labeled K1-K8, with magnetic media $(\mathrm{Cr}, 150 \mathrm{~nm} / \mathrm{CoCrNi}, 50 \mathrm{~nm})$ on aluminum substrates and covered with amorphous carbon overcoats were used in this study. Plated NiP on aluminum substrate disks were mechanically polished to an $R_{\mathrm{a}}$ value of about 4 $\mathrm{nm}$. The carbon overcoats with a nominal thickness of $30 \mathrm{~nm}$ were sputtered over the NiP using an Ulvac-SIH-S 100 in-line system with a base pressure of $2 \times 10^{-8}$ Torr. All sputtering was performed with d.c. power applied to the cathode. Different deposition conditions, i.e. varied bias voltage, substrate temperature, Ar gas pressure and sputter power, were used to obtain a wide range of Raman spectra. The detailed deposition conditions are shown in Table 1 . The Raman spectra were collected with an instrument especially designed for diamond and diamond-like carbon materials. The system comprised a Spex $1250 \mathrm{M}$ spectrometer having a focal length of $1.25 \mathrm{~m}$ and an ISA multichannel CCD detector. The spectrometer housed a 600 groove $\mathrm{mm}^{-1}$ grating component which produced a spectral resolution of approximately $5 \mathrm{~cm}^{-1}$. The spectra were collected with the $514.5 \mathrm{~nm}$ laser line focused by the microprobe to a spot size approximately $3 \mu \mathrm{m}$ in diameter. The Raman spectrum of sample $\mathrm{K} 1$ is shown in Fig. 1. Parameters such as position and intensity of the $G$ and $D$ bands were obtained by fitting the Raman spectrum with two Gaussian distributions and one linear background inten-

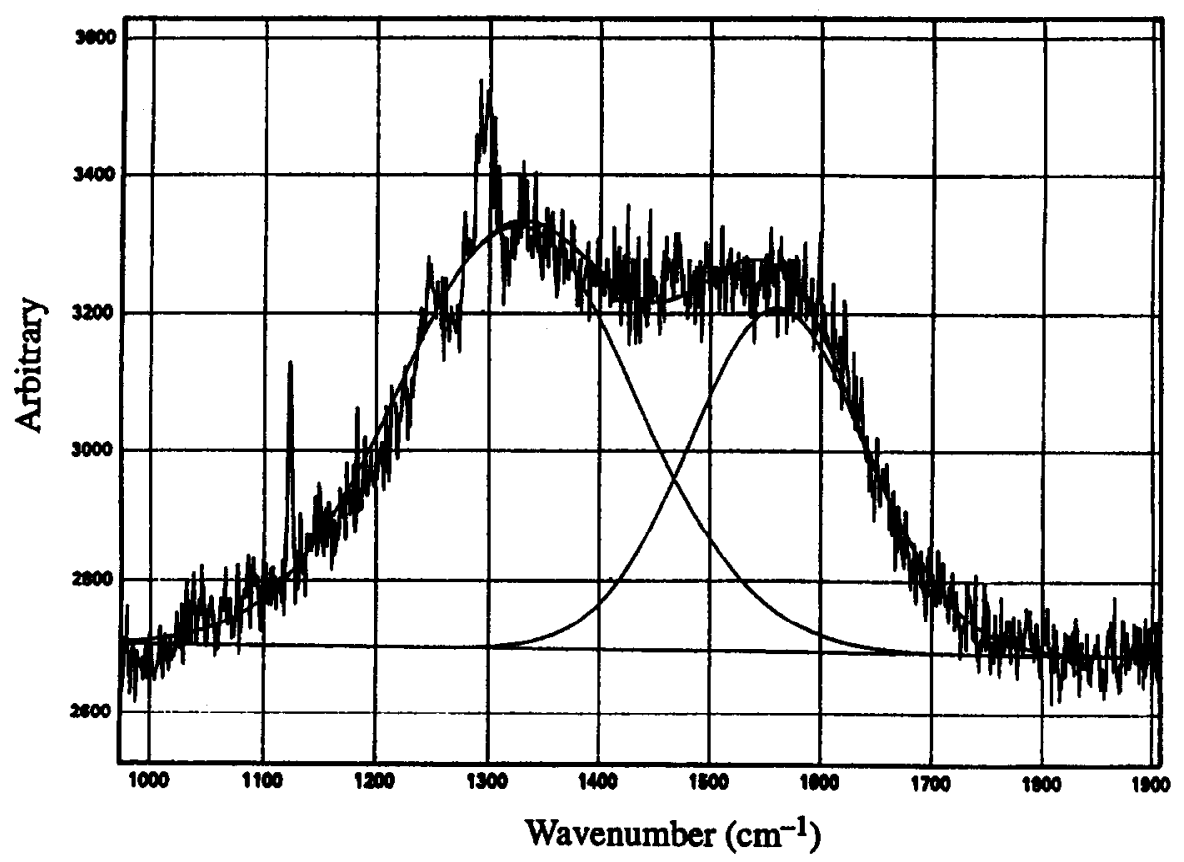

Fig. 1. Typical Raman spectrum of amorphous carbon. 
Table 2

Peak positions and intensity ratios of $\mathrm{G}$ and $\mathrm{D}$ bands for samples $\mathrm{K} 1-\mathrm{K} 8$

\begin{tabular}{lcccccrrr}
\hline & K1 & K2 & K3 & K4 & K5 & K6 & K7 & K8 \\
\hline G-band $\left(\mathrm{cm}^{-1}\right)$ & 1561 & 1578 & 1519 & 1563 & 1573.8 & 1574 & 1582 \\
D-band $\left(\mathrm{cm}^{-1}\right)$ & 1325 & 1353 & 1295 & 1334 & 1349 & 1376 & 1371 \\
$l(\mathrm{D}) / I(\mathrm{G})$ & 1.22 & 1.18 & 1.54 & 1.20 & 1.22 & 1.20 & 1.16 & 1.21 \\
\hline
\end{tabular}

sity. Several spectra were acquired on each sample to ensure that the data were representative of the overall film quality. The results are summarized in Table 2 .

\section{Experimental apparatus and methods}

Fig. 2 shows a schematic diagram of the PCM [14]. The tip assembly, attached to a laminated PZT (lead zirconate titanate) actuator, is made of a diamond tip mounted on a cantilever. The diamond tip is a three-sided pyramid with a nominal tip radius of about $100 \mathrm{~nm}$ and an apex angle of $80^{\circ}$. The sample is mounted on a piezoelectric tube scanner, which can move in the $x, y$ and $z$ directions. The $z$-displacement of the tip is controlled through the voltage applied to the laminated PZT actuator. A focus-error-detection optical head with a resolution better than $1 \mathrm{~nm}$ is used to measure the deflection of the cantilever. The PCM is connected through an interface circuit to a Digital Instruments Nanoscope II (DI NSII) SPM controller, and it uses the control and display software of this instrument.

To make an indentation using the PCM, the tube scanner power supplies are first adjusted such that the tip touches the sample with a small scanning force. The laminated PZT power supply voltage is then changed by a calculated amount to affect the desired normal displacement of the base of the cantilever. Since a feedback controller is used to control the $z$-displacement of the tube scanner such that the tip displacement is a constant, the indentation force can be calculated from the equation:

load $=$ spring constant $\times \Delta z+$ scanning force

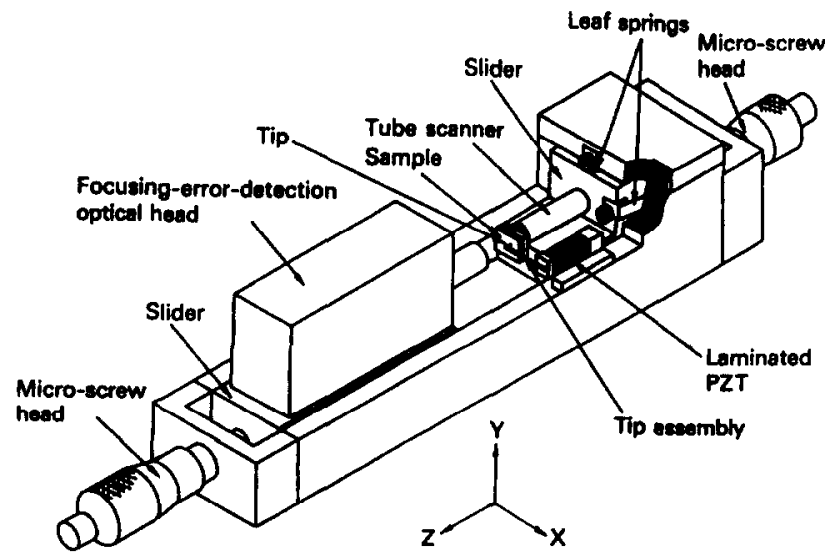

Fig. 2. Schematic diagram of the point contact microscope [14]. where $\Delta z$ is the displacement of the PZT actuator. After indentation, $\Delta z$ is reset to zero and the PCM is controlled by the NSII controller to scan the sample surface for image acquisition. The hardness is defined as the ratio of the indentation force to the projected area of the residual indentation. A detailed description of the indentation hardness tests has been given in Ref. [11].

The PCM can also be used to perform nano-wear tests by the following procedure. A light force is used to scan a surface area to obtain the surface topography. This surface image provides a reference for detecting the wear depth. Then, a heavier load, which is large enough to cause wear, is applied to the tip while the sample raster scans under the tip. Several tests are necessary to find the appropriate conditions under which the wear resistance of different samples can be clearly distinguished. After scratching with the heavy load, the applied load and scan size are reset to the original values to measure the damaged surface. There are two indicators for the characterization of the wear resistance of the layered media. Wear depths are convenient for comparing the wear resistances of carbon overcoats. On the other hand, the critical cycle, at which the overcoat is suddenly broken through, indicates the layer-substrate adhesion strength [15]. This nano-wear test has also been successfully applied by Miyamoto et al. [16] on $\mathrm{C}^{+}$-implanted silicon. The results of both nano-indentation and nano-wear tests depend on the tip used $[11,15]$. In order to compare the characterizations of different overcoats, the same tip was used for all the tests.

\section{Mechanical properties by the PCM}

The PCM was used to perform indentation hardness and nano-wear tests on the samples using a diamond indenter with a tip radius of $100 \mathrm{~nm}$. Table 3 summarizes the results, where hardness was measured at indentation depths of about $5 \mathrm{~nm}$. The nano-wear test was used to identify wear resistance under the following test conditions: scan size, $2 \mu \mathrm{m}$; scan rate, $1.93 \mathrm{~Hz}$; and scan pitch, $10 \mathrm{~nm}$; while three different

Table 3

Hardness values and critical forces for samples K1-K8

\begin{tabular}{|c|c|c|c|c|c|c|c|c|}
\hline & K1 & K2 & K3 & K4 & K5 & K6 & K7 & K8 \\
\hline $\begin{array}{l}\text { Hardness } \\
\text { (GPa) }\end{array}$ & 14 & 18 & 25 & 21 & 16 & 23 & 14 & 27 \\
\hline $\begin{array}{l}\text { Critical force } \\
(\mu N)\end{array}$ & 137 & 118 & 167 & 196 & 137 & 206 & 147 & 216 \\
\hline
\end{tabular}



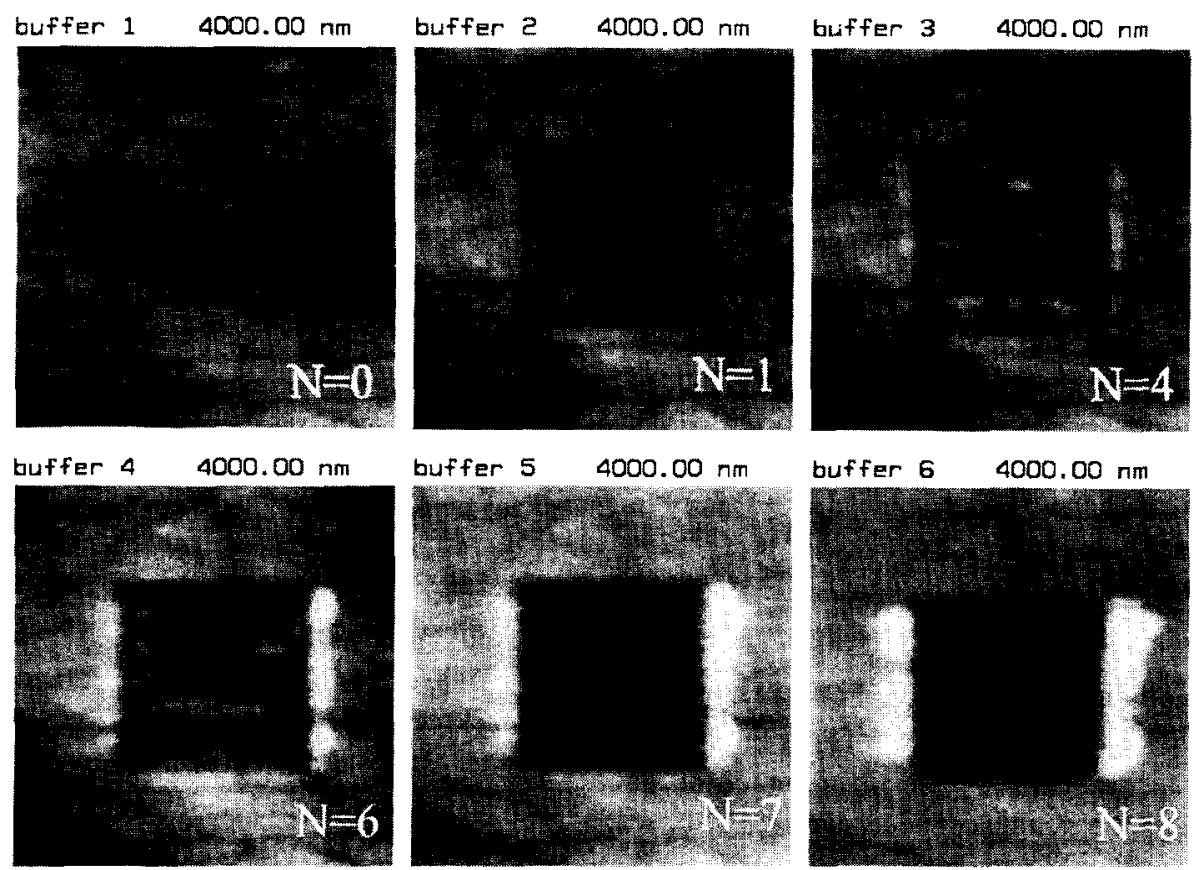

Fig. 3. Evolution of wear mark on sample K3 with cycle number.
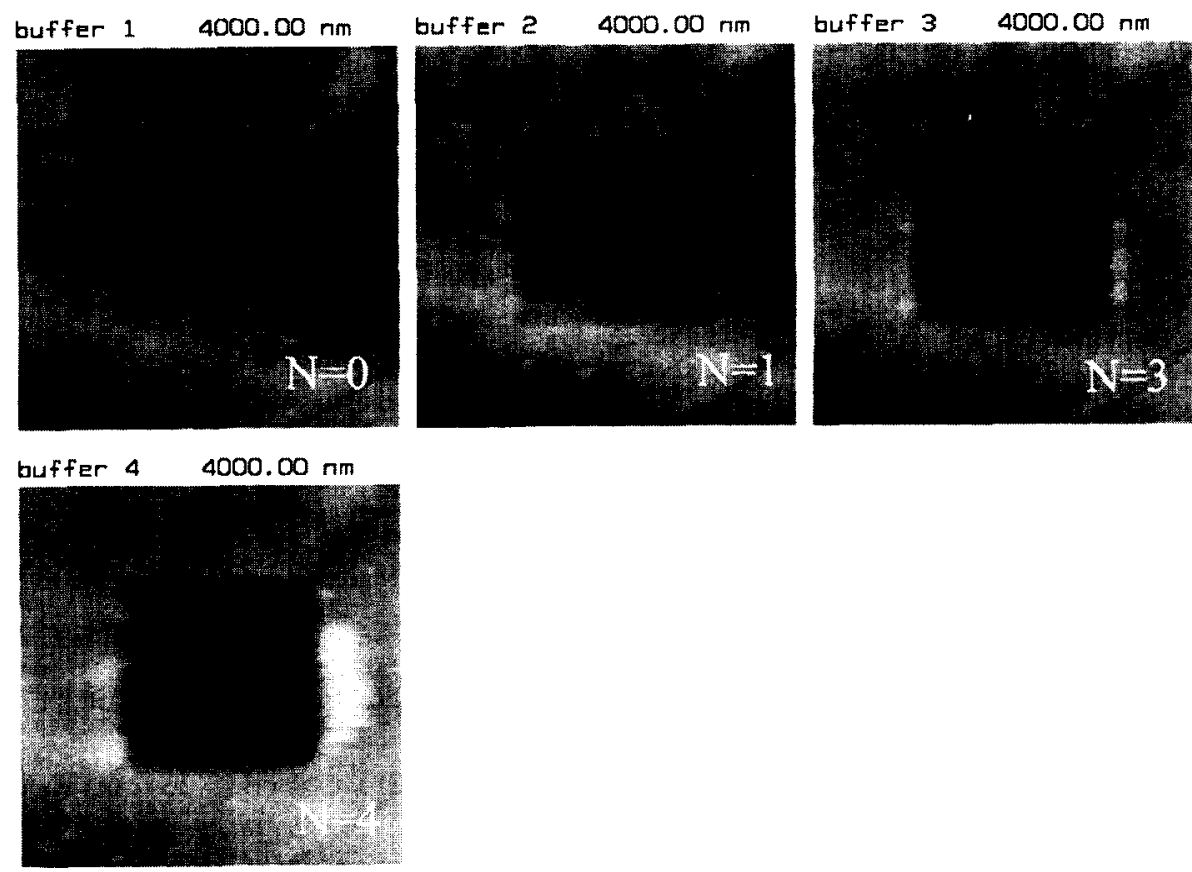

Fig. 4. Evolution of wear mark on sample $\mathrm{K} 5$ with cycle number.

loads of 99,122 and $158 \mu \mathrm{N}$ were applied. Fig. 3 shows a series of wear marks on sample $\mathrm{K} 3$ at different wear cycles with a load of $99 \mu \mathrm{N}$, where $N$ is the cycle number. At the first cycle, there was a wear mark about $3 \mathrm{~nm}$ deep (buffer 2). After three more wear cycles, the wear mark became a little deeper and some debris, shown as white pile-ups inside and around the wear mark (buffer 3 ) began to appear. Two wear cycles later, the overcoat was worn through at two weak portions, as indicated by the two black holes in buffer 4 . The wear depth at the overcoat-broken part is about $35 \mathrm{~nm}$, while at the remaining part the wear depth is about $6 \mathrm{~nm}$. After the next cycle, the overcoat was totally removed (buffer 5 ). The wear test on sample $\mathrm{K5}$ with a load of $99 \mu \mathrm{N}$, is shown in Fig. 4 as another example. Comparing Fig. 4, buffers 2 and 3 , we can see the suddenly worn away portion of the carbon overcoat. Another feature to point out is that the breakthrough of the overcoat usually, although not always, initially occurs at the intersection of two texture lines, as can be seen easily from Fig. 4, buffer 2. For sample K3 (Fig. 3), the initial breakthrough points of the overcoat are also located at the intersection points of texture lines as can be seen by comparing buffers 2 and 3. 


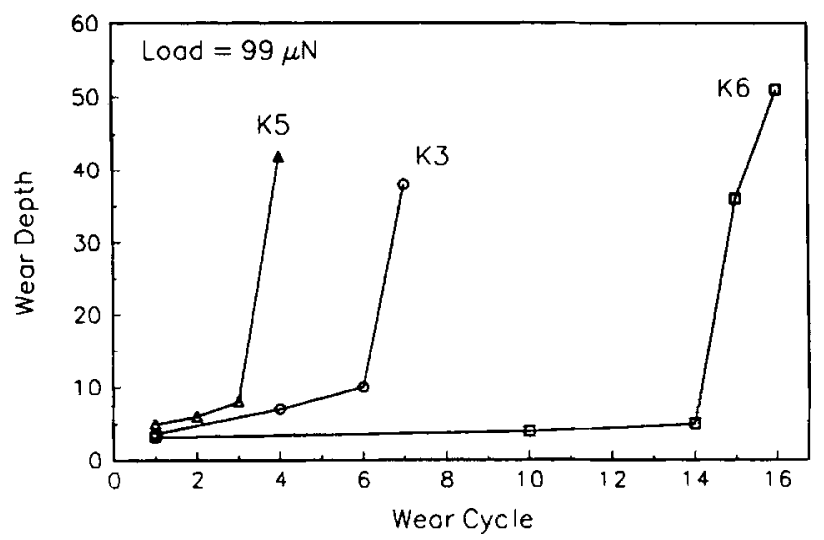

Fig. 5. Wear depth vs. wear cycle for samples K3, K5 and K6.

Table 4

Critical cycles at various loads for samples K1-K8

\begin{tabular}{rrrrrrrrr}
\hline Load $(\mu \mathrm{N})$ & $\mathrm{K} 1$ & $\mathrm{~K} 2$ & $\mathrm{~K} 3$ & $\mathrm{~K} 4$ & $\mathrm{~K} 5$ & $\mathrm{~K} 6$ & $\mathrm{~K} 7$ & K8 \\
\hline 99 & 4 & 2 & 6 & 13 & 3 & 17 & 4 & 23 \\
122 & 3 & 1 & 3 & 7 & 2 & 6 & 2 & 8 \\
158 & 2 & 1 & 2 & 5 & 1 & 3 & 1 & 5 \\
\hline
\end{tabular}

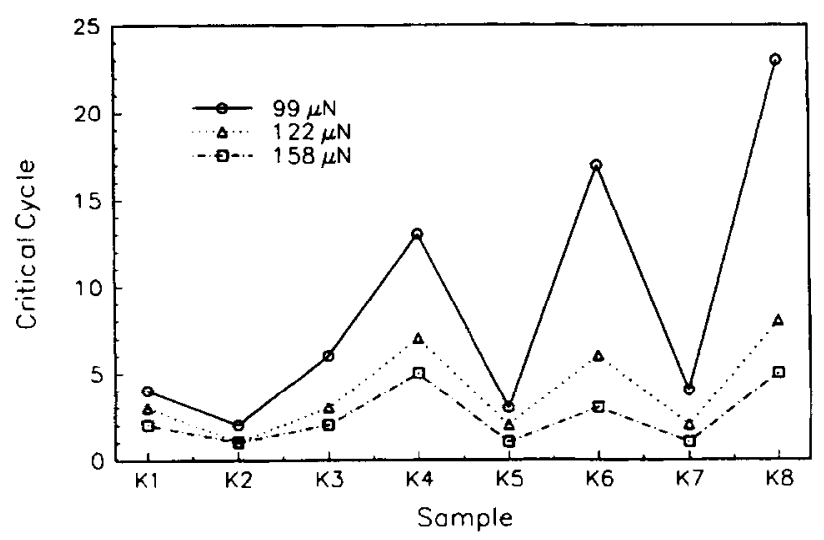

Fig. 6. Critical cycle at varius loads for samples K1-K8.

Fig. 5 shows three typical curves of wear depth versus cycle number for samples $\mathrm{K} 3, \mathrm{~K} 5$ and $\mathrm{K} 6$ under a loading force of $99 \mu \mathrm{N}$, where the wear depth is the mean value over the worn mark. A common feature for these curves is that there exists a break point, the critical cycle, dividing the curves into two regimes. Initially, the wear rate is small; after the critical cycle, it abruptly becomes much larger. The wear depth before the critical cycle is much less than the overcoat thickness. On the other hand, the wear depths at the overcoatbroken points are greater than the overcoat thickness. Therefore, the critical cycle is an indicator of the film-substrate adhesion strength.

Three loading forces, 99,122 and $158 \mu \mathrm{N}$, were used to study the changes in the critical cycle with load. Table 4 summarizes critical cycles at various loads. For clarity, the results are also plotted in Fig. 6 . The critical cycle decreases with increasing load, as expected. The critical cycles of the samples cover a wide range and hence can be used effectively to discriminate differences in performance. The differences in the critical cycles between the samples decrease with increasing load, therefore a suitable load should be used in order to distinguish different samples.

Another method to mcasurc the adhesion strength between the overcoat and the substrate is to find the critical force, defined as the minimum force required to break through the overcoat at the first wear cycle. The critical forces for samples $\mathrm{K} 1$ through $\mathrm{K} 8$ are listed in Table 3. This measure of wear resistance can be compared with the critical cycle. Fig. 7 shows the critical force versus critical cycle curve (data from Table 3 and Table 4), where the critical cycle is obtained under the loading force of $99 \mu \mathrm{N}$. Critical cycle increases with critical force. The slope of the curve decreases with the critical cycle and approaches zero at large loading forces. This is due to the fact that with a strong film-substrate adhesion, i.e. a large critical load, the loading force used to find the critical cycle, in this case $99 \mu \mathrm{N}$, is not enough to initiate the interfacial failure.

As can be seen from Fig. 5, in order to characterize the wear resistance of the overcoats without the effect of the overcoat-substrate adhesion strength, the wear depth should be much less than the thickness of the overcoat. Here, the wear depth at one wear cycle under the loading force of 99 $\mu \mathrm{N}$ is used as an indicator for the wear resistance of the overcoat. The first cycle wear depths of the samples are summarized in Table 5. Fig. 8 shows the first cycle wear depths and the inverse of hardness values of the samples. Each point represents the average of four tests. For sample K2, the overcoat was sometimes worn away at the first cycle. Therefore, the result of $\mathrm{K} 2$ is excluded from the wear depth curve. The wear depth and the inverse of hardness curves present a strong correlation. Consequently, hardness is an important factor in

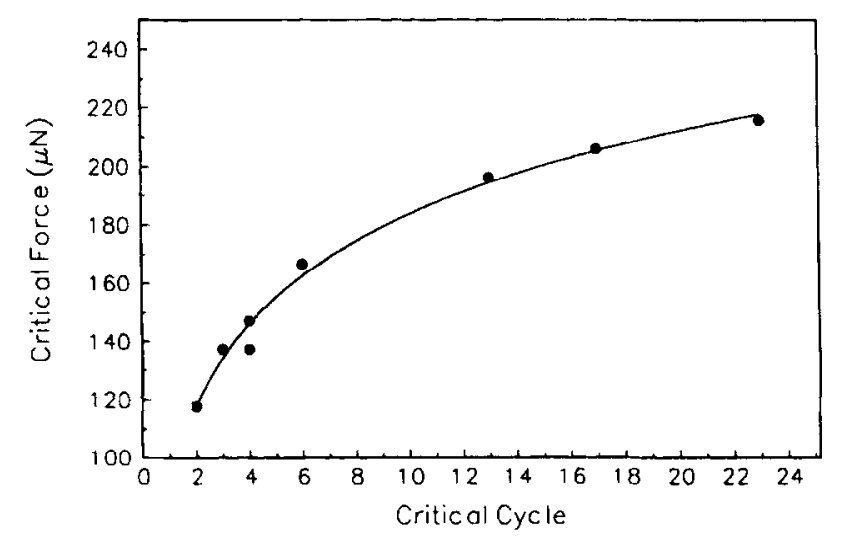

Fig. 7. Relationship between critical force and critical cycle.

Table 5

First-cycle wear depths at various loads for samples K1-K8

\begin{tabular}{lcccccccc}
\hline $\operatorname{Load}(\mu \mathrm{N})$ & $\mathrm{K} 1$ & $\mathrm{~K} 2$ & $\mathrm{~K} 3$ & $\mathrm{~K} 4$ & $\mathrm{~K} 5$ & $\mathrm{~K} 6$ & $\mathrm{~K} 7$ & $\mathrm{~K} 8$ \\
\hline 99 & 3 & 12 & 2.6 & 2.2 & 4.1 & 2.7 & 5.2 & 2 \\
122 & 4.7 & 38 & 4.6 & 4.2 & 5.8 & 3.9 & 7.6 & 3.6 \\
158 & 40 & 45 & 41 & 4.8 & 49 & 5.1 & 58 & 5.9 \\
\hline
\end{tabular}




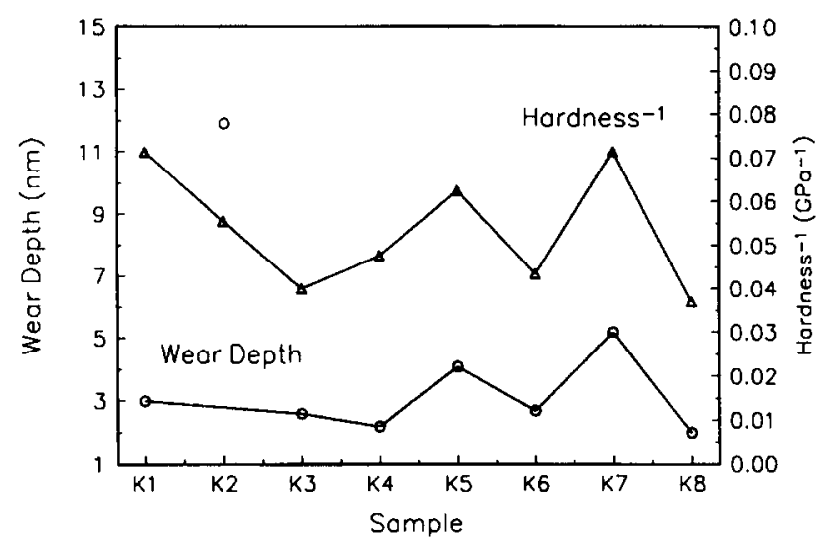

Fig. 8. Correlation between the first-cycle wear depth and hardness.
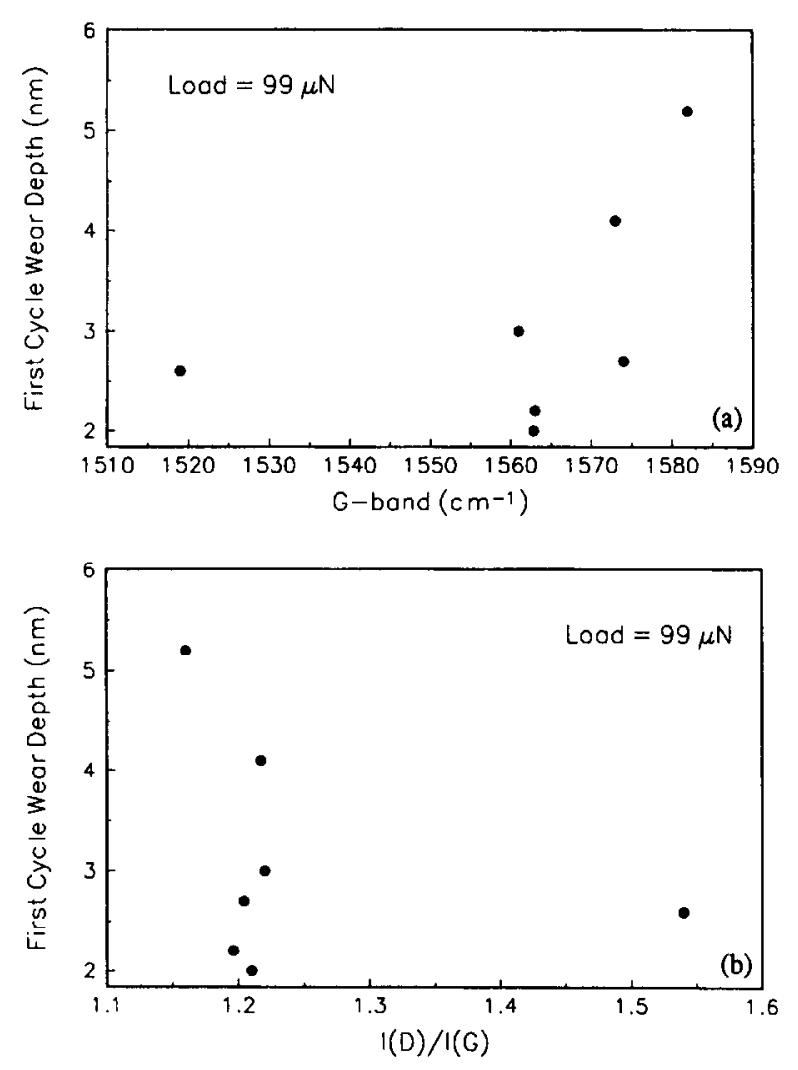

Fig. 9. First-cycle wear depth vs. (a) G-band and (b) $I(\mathrm{D}) / I(\mathrm{G})$ ratio.

the wear resistance and a high hardness value usually implies small wear depth.

\section{Comparison and discussion}

The first cycle wear depth under a loading force of $99 \mu \mathrm{N}$ is plotted as a function of the G-band position and $I(\mathrm{D}) /$ $I(\mathrm{G})$ ratio in Fig. 9(a) and 9(b), respectively. The result for sample $\mathrm{K} 2$ is excluded for the same reason discussed in the previous section. The lack of data in the G-band ranging from 1525 to $1555 \mathrm{~cm}^{-1}$ and $I(\mathrm{D}) / I(\mathrm{G})$ ratio ranging from 1.25 to 1.5 makes it difficult to have a complete picture of the relation between wear depth and G-band position or $I(D) /$ $I(G)$ ratio. However, Fig. 9(a) appears to present a correla- tion between the shift of the G-band to a higher frequency and poorer wear resistance. Fig. 9 (b) shows the same feature: the data scatters in a small range around the $I(\mathrm{D}) / I(\mathrm{G})$ ratio of about 1.2, but a tendency is suggested, considering the larger range. The average of wear depths of the five points around $I(\mathrm{D}) / I(\mathrm{G})$ of about 1.2 is $2.8 \mathrm{~nm}$, which lies between the wear depths of the remaining two points. Therefore, a higher $I(\mathrm{D}) / I(\mathrm{G})$ ratio may imply a better wear resistance. This result agrees with that of Marchon et al. [8] regarding the relation between the abrasive wear rate and $I(\mathrm{D}) / I(\mathrm{G})$ ratio. As discussed in the previous section, there is a strong correlation between first-cycle wear depth and hardness. Therefore, it can be deduced that a similar relation also exists between hardness and G-band position and/or $I(\mathrm{D}) / I(\mathrm{G})$ ratio.

Fig. 10(a) and 10(b) show critical force versus G-band position and $I(\mathrm{D}) / I(\mathrm{G})$ ratio, respectively. As can be seen from the figures, the data points scatter along the whole range. Consequently, critical force is independent of either G-band position or $I(\mathrm{D}) / I(\mathrm{G})$ intensity ratio.

It is generally believed that the downshift of the G-band may imply an increase in the $\mathrm{sp}^{3} / \mathrm{sp}^{2}$ ratio and therefore an increase of hardness and/or wear resistance. However, an increase in $\mathrm{sp}^{3} / \mathrm{sp}^{2}$ ratio is not a necessary condition for the downshift of the G-band position. For example, residual tensile stress of carbon films may also induce a downshift of the G-band [17] while producing a decrease in hardness [18]. Therefore, a low G-band frequency does not necessarily
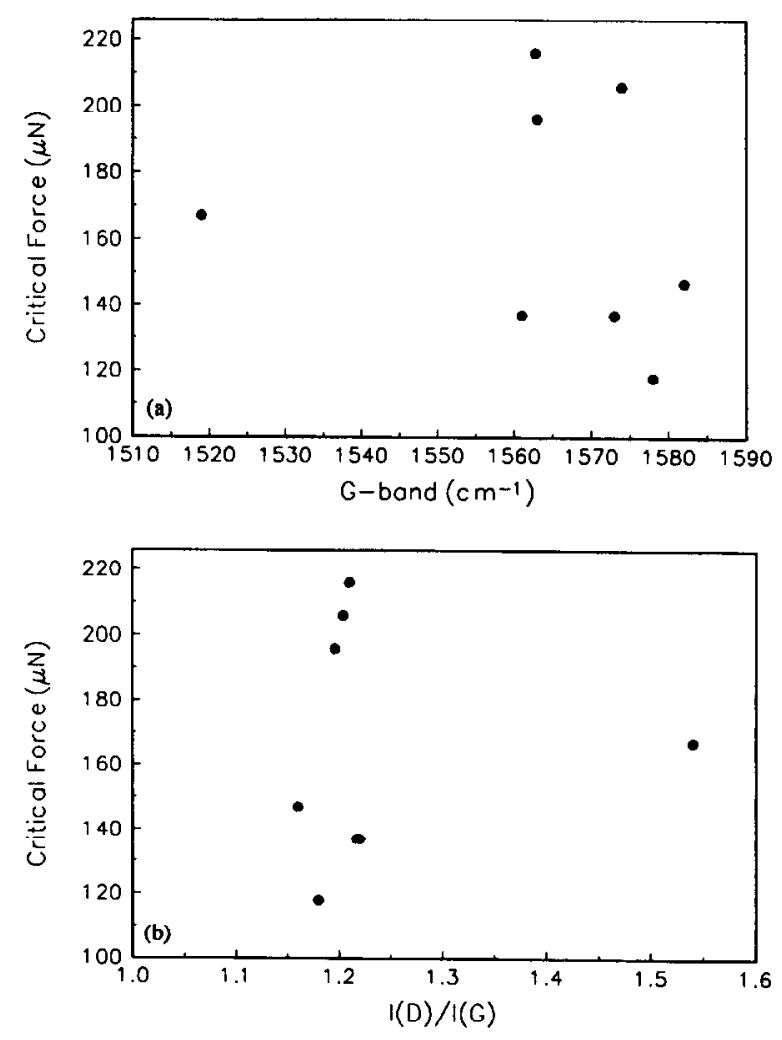

Fig. 10. Relationship between critical force and (a) G-band and (b) $l$ (D)/ $I(\mathbf{G})$ ratio. 
imply high wear resistance. This could possibly be used to explain the scatter of data points in Fig. 9(a).

In polycrystalline graphite, the $I(\mathrm{D}) / I(\mathrm{G})$ ratio describes the microcrystallite size. For amorphous carbon, a large $I(\mathrm{D}) / I(\mathrm{G})$ may indicate a large number of small graphite domains embedded in a three-dimensional network of $\mathrm{sp}^{3}$ and $\mathrm{sp}^{2}$-bonded carbon atoms [8]. These small graphite domains may block the movement of dislocations and therefore increase the hardness. This effect may be used to explain the tendency shown in Fig. 9(b): a larger $I(\mathrm{D}) / I(\mathrm{G})$ ratio implies better wear resistance. However, this may not be the only reason for the change in the $I(\mathrm{D}) / I(\mathrm{G})$ ratio. Therefore, carbon films with similar $I(\mathrm{D}) / I(\mathrm{G})$ ratio values may have very different wear resistances.

The critical force and critical cycle describe the adhesion strength between the overcoat and the underlying magnetic layer, which is not an intrinsic property of the carbon overcoat. In this case, we do not expect a strong correlation to exist between Raman spectra and the critical force/cycle.

\section{Summary and conclusion}

The nano-wear test of a layered medium measures the combination of the wear resistance of the overcoat and the overcoat-substrate adhesion strength. The real failure mechanism depends on the relative strengths of these two factors. For a thin film magnetic disk, consisting of a hard overcoat on a soft magnetic layer, breakthroughs of the layer under a fixed load occur even when the wear depth is much less than the layer thickness. The wear cycle at which sudden breakthrough occurs is defined as the critical cycle. At this stage, relatively deep holes with depths close to the layer thickness can be seen inside the wear mark. Here, the major failure mechanism is the breaking of the layer-substrate interface. To characterize the wear resistance of the layer, the wear depth should be measured before the critical cycle, which is usually only a few cycles at this stage. The critical cycle, which can be used to identify the adhesion strength between the layer and the substrate, decreases with increasing load. To obtain a clear distinction between critical cycles for different media, the applied load should be chosen properly. Besides the critical cycle, the minimum force required to totally remove the layer in one cycle, called the critical force, can also be used to measure the layer-substrate adhesion strength. There is a strong correlation between the critical force and the critical cycle.

Besides the sudden collapse of the overcoat described in the above paragraph, it is also found that breakthrough of the overcoat usually occurs at the junctions of texture lines. There may be some initial cracks or high stress concentration at the texture-line junctions which make it vulnerable to wear. Hence, texture lines, which are necessary for the reduction of the stiction force between the slider and the disk surface during the contact start process, may weaken the wear durability of the system.
There exists a certain relation between the Raman spectrum and the mechanical properties of carbon overcoats. It is generally believed that a high $\mathrm{sp}^{3} / \mathrm{sp}^{2}$ bonding ratio correlates well with the G-band shift to a lower frequency and an increase in diamond-like structures may increase the wear resistance and hardness. In addition, an increase in the $I(\mathrm{D}) /$ $I(\mathrm{G})$ intensity ratio may result from an increase in the number of small graphite domains embedded in a three-dimensional network of carbon atoms and/or an increase of edge density, which may block the movement of dislocations and hence increase the wear resistance. These reasons can be used to explain some correlations between the mechanical properties of carbon overcoats and their Raman spectra. However, there are factors other than bonding ratio and the edge density that will also induce changes in Raman spectra. In addition, physical properties may not solely depend on the bonding ratio and crystallite size. Therefore, carbon overcoats having similar values of G-band frequency and/or $I(\mathrm{D}) / I(\mathrm{G})$ ratio may have different mechanical properties. The Raman spectrum is helpful for the study of microstructure of carbon overcoats, but it cannot be used as the sole indicator for the wear performance of carbon overcoats.

\section{Acknowledgements}

This research was supported by the Computer Mechanics Laboratory at the University of California at Berkeley and Kobe Steel USA.

\section{References}

[1] T.J. Dines, D. Either, A. Dehbi and A. Mathews, Raman spectra of hard carbon films and hard carbon films containing secondary elements, Carbon, 29(2) (1991) 225-231.

[2] M. Nakamize, R. Kammereck and P.L. Walker, Laser Raman studies on carbons, Carbon, 12 (1973) 259-267.

[3] M. Yoshikawa, N. Nagai, M. Mastsuki, H. Fukuda, G.H. Katagiri, Ishida, A. Ishitani and I. Nagai, Raman scattering from $s p^{2}$ carbon clusters, Phys. Rev. B, 46(11) (1992) 7169-7174.

[4] Y. Wang, D. Alsmeyer and R. McCreery, Raman spectroscopy of carbon materials: structural basis of observed spectra, Chem. of Mater., 2 (1990) 557-563.

[5] D. Beeman, J. Silverman, R.Lynds and M.R. Anderson, Modeling studies of amorphous carbon, Phys. Rev. B, 30(2) (1984) 870-875.

[6] F. Tuinstra and J.L. Koenig, Raman spectrum of graphite, J. Chem. Phys., 53(3) (1970) 1126-1130.

[7] D.S. Knight and W.B. White, Characterization of diamond films by Raman spectroscopy, J. Mater. Res., 4(2) (1989) 385-393.

[8] B. Marchon, N. Heiman, M. R, Khan, A. Lauite, J.W. Ager III and D.K. Veirs, Raman and resistivity investigation of carbon overcoats of thin-film media: correlation with tribological properties, J. Appl. Phys., 69 ( 1991 ) 5748-5750.

[9] J.L. Lauer and L. DuPlessis, Relation between deposition parameters, structure and Raman spectra of carbon overcoats on simulated magnetic storage disks, STLE Tribol. Trans., 36(1) (1993) 19-26. 
[10] E.H. Lee, D.M. Hembree, G.R. Rao and L.K. Mansur, Raman scattering from ion-implanted diamond, graphite and polymers, $P$ hys. Rev. B, 48(21) (1993) 15540-15551.

[11] C.-J. Lu, D.B, Bogy and R. Kaneko, Nanoindentation hardness tests using a point contact microscope, ASME J. Tribol., 116 (1994) 175180.

[12] C.-J. Lu and D. Bogy, Sub-microindentation hardness tests on thin film magnetic disks, ASME Adv. Info. Storage Systems, 6 (1995) 163175.

[13] Z. Jiang, C.-J. Lu, D.B. Bogy, C.S. Bhatia and T, Miyamoto, Nanotribological characterization of hydrogenated carbon films by scanning probe microscopy, Thin Solid Films, 258(1-2) (1995) 7581.
[14] T. Miyamoto, R. Kaneko and S. Miyake, Tribological characteristics of amorphous carbon films investigated by point contact microscopy, J. Vac. Sci. Technol., B, 9(2) (1991) 1336-1339.

[15] Z. Jiang, C.-J. Lu, D.B. Bogy and T. Miyamoto, An investigation on experimental conditions and characteristics of a nano-wear test, Wear, 181-183(2) (1995) 777-783.

[16] T. Miyamoto, S. Miyake and R. Kaneko, Wear resistance of $\mathrm{C}^{+}$. implanted silicon investigated by scanning probe microscopy, Wear, 162 (1993) 733-738.

[17] M. Kitajima and K. Nakamura, Evaluation of local stress on carbon materials by Raman spectroscopy, J. Nucl. Mater., 175(3) (1990) 251-253.

[18] J. Frankel, A. Abbate and W. Scholz, The effect of residual stresses on hardness measurements, Exp. Mech., 33(2) (1993) 164-168. 